\title{
A novel approach for absolute radar calibration: formulation and theoretical validation
}

\author{
C. Merker ${ }^{1}$, G. Peters ${ }^{3}$, M. Clemens ${ }^{1}$, K. Lengfeld ${ }^{1}$, and F. Ament ${ }^{1,2}$ \\ ${ }^{1}$ Meteorological Institute of the University of Hamburg, Hamburg, Germany \\ ${ }^{2}$ Max Planck Institute for Meteorology, Hamburg, Germany \\ ${ }^{3}$ METEK Meteorologische Messtechnik GmbH, Elmshorn, Germany \\ Correspondence to: C. Merker (claire.merker@uni-hamburg.de)
}

Received: 20 January 2015 - Published in Atmos. Meas. Tech. Discuss.: 05 February 2015

Revised: 04 June 2015 - Accepted: 06 June 2015 - Published: 22 June 2015

\begin{abstract}
The theoretical framework of a novel approach for absolute radar calibration is presented and its potential analysed by means of synthetic data to lay out a solid basis for future practical application. The method presents the advantage of an absolute calibration with respect to the directly measured reflectivity, without needing a previously calibrated reference device. It requires a setup comprising three radars: two devices oriented towards each other, measuring reflectivity along the same horizontal beam and operating within a strongly attenuated frequency range (e.g. K or X band), and one vertical reflectivity and drop size distribution (DSD) profiler below this connecting line, which is to be calibrated. The absolute determination of the calibration factor is based on attenuation estimates.

Using synthetic, smooth and geometrically idealised data, calibration is found to perform best using homogeneous precipitation events with rain rates high enough to ensure a distinct attenuation signal (reflectivity above ca. $30 \mathrm{dBZ}$ ). Furthermore, the choice of the interval width (in measuring range gates) around the vertically pointing radar, needed for attenuation estimation, is found to have an impact on the calibration results.

Further analysis is done by means of synthetic data with realistic, inhomogeneous precipitation fields taken from measurements. A calibration factor is calculated for each considered case using the presented method. Based on the distribution of the calculated calibration factors, the most probable value is determined by estimating the mode of a fitted shifted logarithmic normal distribution function. After filtering the data set with respect to rain rate and inhomogeneity and choosing an appropriate length of the considered
\end{abstract}

attenuation path, the estimated uncertainty of the calibration factor is of the order of 1 to $11 \%$, depending on the chosen interval width. Considering stability and accuracy of the method, an interval of eight range gates on both sides of the vertically pointing radar is most appropriate for calibration in the presented setup.

\section{Introduction}

In many domains, for example in weather prediction, nowcasting, or hydrology, accurate rainfall monitoring is an ongoing issue. Accurate rain rate estimates can be obtained by using rain gauges, which are continuously measuring at one point and have achievable measurement uncertainties of about $5 \mathrm{~mm} \mathrm{~h}^{-1}$, down to $5 \%$ for precipitation above $100 \mathrm{~mm} \mathrm{~h}^{-1}$ (Vuerich et al., 2009). Even a dense network of these devices only provides point measurements which are not able to describe the high temporal and spatial variability of rainfall events (e.g. Peleg et al., 2013; Pedersen et al., 2010a; Krajewski et al., 2003). Radar networks, such as those from national weather services or smaller ones operated by research institutions, can provide spatially and temporally highly resolved, area-covering rainfall data (e.g. Lengfeld et al., 2014; Trabal et al., 2013; Lim et al., 2011; McLaughlin et al., 2009; Salazar et al., 2009; Chandrasekar and Jayasumana, 2001; Crum et al., 1998) and are already used to improve drainage control and flash-flood warning systems (e.g. Picciotti et al., 2013; Quintero et al., 2012; Delrieu et al., 2009; Corral et al., 2008; Einfalt et al., 2004; Creutin and Borga, 2003; Krajewski and Smith, 2002). However, a disad- 
vantage of using weather radar data is the lower accuracy of the retrieved rain rate in comparison to measurements from rain gauges, since rain rates are not measured directly but derived from reflectivity measurements. Beside errors induced by $Z-R$ relations, attenuation, noise, ground clutter, blocking or interferences, one major limiting factor for precise rain rate determination is radar calibration (e.g. Villarini and Krajewski, 2010; Einfalt et al., 2004; Hunter, 1996). Although recent weather radars making use of polarimetric variables for precipitation estimates are more accurate and not affected by calibration, polarimetric methods are not applicable in all conditions, and a demand for absolute calibration remains.

For relative radar calibration it is common practice to compare reflectivity measurements from radars that simultaneously monitor the same rainfall event (e.g. Hunter, 1996). This adjustment only works for radars within a network and do not allow for an absolute comparison of data with other networks or instruments. Furthermore, the retrieved rain rate cannot be used quantitatively. A frequently used approach is a calibration with respect to rain rate using point measurements from rain gauges (e.g. Sebastianelli et al., 2013; Thorndahl and Rasmussen, 2012; Pedersen et al., 2010b; Jensen and Pedersen, 2005) or disdrometers (e.g. Nielsen et al., 2013; Lee and Zawadzki, 2006) at ground level. This implies the disadvantages of point-to-area comparison and differences in measuring height (e.g. Gires et al., 2014; Jaffrain and Berne, 2012a; Moreau et al., 2009). Additionally, the obtained calibration is highly dependent on the chosen $Z-R$ relation (e.g. Ulbrich and Lee, 1999). In order to avoid this source of error, calibration with respect to radar reflectivity is preferable.

Vertically pointing micro rain radars (MRRs), using a frequency modulated continuous wave (FM-CW) measuring principle and operating at $\mathrm{K}$ band $(24.1 \mathrm{GHz}, \lambda=12.4 \mathrm{~mm})$ (Peters et al., 2002), allow a comparison of data at same height levels, but compared measuring volumes are still not necessarily equal, and micro rain radars also lack an absolute calibration (e.g. van Baelen et al., 2009).

A novel method for absolute calibration using a setup of three radars, performed without previously calibrated reference device, and calibrating with respect to reflectivity is presented here. It requests a radar network setup and takes advantage of the attenuation, which is generally seen as perturbing effect on measurements. The aim of this paper is the theoretical formulation and the proof of concept validation of the presented method, which has not been investigated before.

The analysis of this absolute calibration method focuses on the application on MRRs. Nevertheless, considered instrumental setup and theoretical framework of the method presented in Sect. 2 are applicable to any strongly attenuated radar type. A proof of concept validation is realised in Sect. 3, and validity and potential of the method are analysed further by means of synthetic data presenting realistic measurement structures in Sect. 4. From this study, criteria

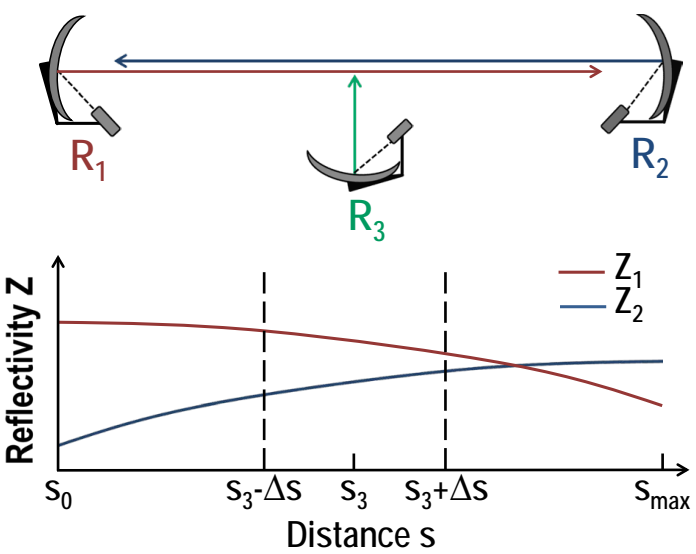

Figure 1. Schematic network setup including horizontally oriented radars $R_{1}$ and $R_{2}$ and vertically oriented radar $R_{3}$ (top panel) and schematic reflectivity measurements for $R_{1}$ and $R_{2}$ along the connecting line (bottom panel).

of appropriated rainfall events for calibration are worked out for subsequent utilisation.

\section{Theoretical framework}

The network setup required in order to apply the absolute calibration method presented here is depicted schematically in Fig. 1. Two horizontally oriented radars $\left(R_{1}\right.$ and $\left.R_{2}\right)$ measure along the same connecting line from opposite directions at a certain height. A third, drop size distribution (DSD) profiling device $\left(\mathrm{R}_{3}\right)$ is positioned below the measuring path in order to provide measurements at one point of the connecting line. For the sake of simplicity, the focus of the study presented in the following is on the calibration of $R_{3}$. However, it is straightforward to calibrate $R_{1}$ and $R_{2}$ once $R_{3}$ is calibrated.

Considering that the two horizontally oriented radars $R_{1}$ and $\mathrm{R}_{2}$ (Fig. 1) operate at a strongly attenuated frequency (e.g. $\mathrm{K}$ band) and measure on the same path from opposite directions, the measured reflectivity provided at a point $s$ on the connecting line, $Z_{1}(s)$ and $Z_{2}(s)$, can be expressed as follows (e.g. Bringi and Chandrasekar, 2001):

$$
\begin{aligned}
& Z_{1}(s)=C_{1} \cdot Z(s) \cdot \exp \left(-2 \int_{s_{0}}^{s} k\left(s^{\prime}\right) \mathrm{d} s^{\prime}\right), \\
& Z_{2}(s)=C_{2} \cdot Z(s) \cdot \exp \left(-2 \int_{s}^{s_{\max }} k\left(s^{\prime}\right) \mathrm{d} s^{\prime}\right) .
\end{aligned}
$$

The positions of $\mathrm{R}_{1}$ and $\mathrm{R}_{2}$ are denoted $s_{0}$ and $s_{\max }$, respectively. Similarly, the measured reflectivity provided by the vertically pointing radar $R_{3}$ at a height $z$ is 
$Z_{3}(z)=C_{3} \cdot Z(z) \cdot \exp \left(-2 \int_{0}^{z} k\left(z^{\prime}\right) \mathrm{d} z^{\prime}\right)$.

The measured reflectivity differs from the intrinsic reflectivity $Z(s)$ by the multiplicative calibration factors $C_{1}, C_{2}$ and $C_{3}$ comprising device characteristics and by the two-way attenuation, with $k$ the specific attenuation. The latter is given by

$k(s)=\int_{D_{\min }}^{D_{\max }} N(D, s) \sigma_{\mathrm{e}}(D) \mathrm{d} D$,

where $N(D, s)$ is the drop size distribution (droplet number per unit volume and per unit size interval) dependent on the drop diameter $D$ and $\sigma_{\mathrm{e}}(D)$ the extinction cross section.

The beam of the vertically pointing Doppler radar $R_{3}$ crosses the connecting line at $s_{3}$, which will be called the reference point in the following. At this point, not only the reflectivity but also the discrete DSD $N_{3}\left(D_{j}, s_{3}, h\right)$ can be derived from the measured Doppler spectra according to the method of Atlas et al. (1973), using an analytical relation between drop terminal velocity and drop size in the absence of vertical winds. Because $N$ is proportional to $Z$, the relation between measured and intrinsic DSD is analogous to the expression for the reflectivity:

$N_{3}\left(D_{j}, s_{3}, h\right)=C_{3} \cdot N\left(D_{j}, s_{3}, h\right) \cdot \exp \left(-2 \int_{0}^{h} k\left(z^{\prime}\right) \mathrm{d} z^{\prime}\right)$,

where $h$ is the height of the reference point above $\mathrm{R}_{3}$ and $C_{3}$ the same as in Eq. (2). Now we determine the measured attenuation using Eq. (3) with discrete size classes $D_{j}$ :

$k_{3}\left(s_{3}\right)=\sum_{D_{\min }}^{D_{\max }} N_{3}\left(D_{j}, s_{3}, h\right) \sigma_{\mathrm{e}}\left(D_{j}\right) \Delta D_{j}$.

Here, $\Delta D_{j}$ is the width of the size classes. Replacing the measured DSD $N_{3}\left(D_{j}, s_{3}, h\right)$ by Eq. (4) yields

$$
\begin{aligned}
k_{3}\left(s_{3}\right) & =\sum_{D_{\min }}^{D_{\max }} C_{3} N\left(D_{j}, s_{3}\right) \exp \left(-2 \int_{0}^{h} k\left(z^{\prime}\right) \mathrm{d} z^{\prime}\right) \sigma_{\mathrm{e}}\left(D_{j}\right) \Delta D_{j} \\
& =C_{3} \cdot \exp \left(-2 \int_{0}^{h} k\left(z^{\prime}\right) \mathrm{d} z^{\prime}\right) \cdot \sum_{D_{\min }}^{D_{\max }} N\left(D_{j}, s_{3}\right) \sigma_{\mathrm{e}}\left(D_{j}\right) \Delta D_{j} \\
& =C_{3} \cdot \exp \left(-2 \int_{0}^{h} k\left(z^{\prime}\right) \mathrm{d} z^{\prime}\right) \cdot k\left(s_{3}\right) .
\end{aligned}
$$

In the following, assuming homogeneous conditions in the environment of the reference point allows for resolving Eq. (6) for $C_{3}$ :
$C_{3}=\frac{k_{3}\left(s_{3}\right)}{\exp \left[-2 k\left(s_{3}\right) h\right] \cdot k\left(s_{3}\right)}$.

This implies the necessary condition of a constant specific attenuation in the vertical section between $\mathrm{R}_{3}$ and the height of the measuring path (about 40 to $80 \mathrm{~m}$, depending on the network setup).

While $k_{3}\left(s_{3}\right)$ is known from Eq. (5), $k\left(s_{3}\right)$ is derived by comparing reflectivity measurements from $\mathrm{R}_{1}$ and $\mathrm{R}_{2}$ along a selected section of the measuring path. Again, constant specific attenuation along this particular section is required. Considering the high spatial variability of rainfall on small scales (e.g. Gires et al., 2014; Jaffrain and Berne, 2012b; Mandapaka et al., 2009), one important challenge of the method becomes obvious here. The section bounds are located at $s_{3}-\Delta s$ and $s_{3}+\Delta s$ on both sides of $\mathrm{R}_{3}$. From Eq. (1), the ratio between measured reflectivity at $s_{3}-\Delta s$ and measured reflectivity at $s_{3}+\Delta s$ for each radar $\mathrm{R}_{1}$ and $\mathrm{R}_{2}$ separately yields

$\frac{Z\left(s_{3}-\Delta s\right)}{Z\left(s_{3}+\Delta s\right)}=\frac{Z_{1}\left(s_{3}-\Delta s\right)}{Z_{1}\left(s_{3}+\Delta s\right)} \cdot \exp \left(-4 k\left(s_{3}\right) \Delta s\right)$

and

$$
\frac{Z\left(s_{3}-\Delta s\right)}{Z\left(s_{3}+\Delta s\right)}=\frac{Z_{2}\left(s_{3}-\Delta s\right)}{Z_{2}\left(s_{3}+\Delta s\right)} \cdot \exp \left(4 k\left(s_{3}\right) \Delta s\right) .
$$

Notice that the calibration factors $C_{1}$ and $C_{2}$ cancel out at this point, ensuring the absolute determination of the specific attenuation $k\left(s_{3}\right)$ needed for absolute calibration.

Equalising Eqs. (8) and (9) and rearranging terms gives an expression in which only the specific attenuation $k$ remains as a function of known values:

$\frac{Z_{1}\left(s_{3}-\Delta s\right) \cdot Z_{2}\left(s_{3}+\Delta s\right)}{Z_{1}\left(s_{3}+\Delta s\right) \cdot Z_{2}\left(s_{3}-\Delta s\right)}=\exp \left(8 k\left(s_{3}\right) \Delta s\right)$.

By assuming a constant attenuation factor $k\left(s_{3}\right)$ along the considered section, the latter can then be expressed by

$k\left(s_{3}\right)=\ln \left[\frac{Z_{1}\left(s_{3}-\Delta s\right) \cdot Z_{2}\left(s_{3}+\Delta s\right)}{Z_{1}\left(s_{3}+\Delta s\right) \cdot Z_{2}\left(s_{3}-\Delta s\right)}\right] \cdot \frac{1}{8 \Delta s}$.

Having determined the absolute specific attenuation at $s_{3}$, comparison with the specific attenuation $k_{3}\left(s_{3}\right)$ obtained from DSD measurements of $\mathrm{R}_{3}$ (Eq. 5) allows for absolute calibration of $R_{3}$. Combining Eqs. (7) and (11) yields the equation for the absolute calibration factor $C_{3}$. The total expression for $C_{3}$ now only depends on measured values.

This calibration approach is valid provided that the DSD measured by $\mathrm{R}_{3}$ is representative for the DSD along the measuring path between $s_{3}-\Delta s$ and $s_{3}+\Delta s$. Also, it is worth mentioning that errors in DSD measurements from $\mathrm{R}_{3}$ propagate directly to the obtained calibration factor through the specific attenuation $k_{3}\left(s_{3}\right)$. The main source of errors for DSD when measuring under real conditions with MRRs is 
vertical wind. Considering a study from Peters et al. (2005), specific attenuation can be overestimated by a factor of 2 for $1 \mathrm{~m} \mathrm{~s}^{-1}$ vertical wind, which is substantial. However, an analysis of vertical wind data with $10 \mathrm{~s}$ resolution at a height of $50 \mathrm{~m}$ (Wettermast Hamburg site, Germany) yields a standard deviation of only $0.49 \mathrm{~m} \mathrm{~s}^{-1}$ for rainfall events. This strongly reduces the possible error to a factor of about 1.4. Since convective precipitation events (inducing strong inhomogeneity) are not suited for calibration, the typical vertical wind variance in considered cases, and thus the measuring error, is even lower. Furthermore, when taking into account the measuring volume of the MRR, which also reduces fluctuation, the error in specific attenuation should be very small.

In the following, the inverse of $C_{3}$ is considered, since $C_{3}^{-1}$ is the factor with which data is corrected after calibration.

\section{Proof of concept}

In order verify the theory of the presented method, it is evaluated using synthetic data obtained from a forward model generating reflectivity out of a given rain rate. These data represent perfectly calibrated devices for validation purpose, which means correction factors $C_{1}^{-1}, C_{2}^{-1}$ and $C_{3}^{-1}$ are implicitly set to 1.0 . The measuring path described in Fig. 1 is divided into 31 range gates of $\Delta r=200 \mathrm{~m}$ width each. Simulated data for $\mathrm{R}_{1}$ and $\mathrm{R}_{2}$ are discretised accordingly. After the discretisation, $1 \leq i \leq 31$ describes the range gates along the measuring section, starting at $\mathrm{R}_{1}$. We also define the interval between $s_{3}-\Delta s$ and $s_{3}+\Delta s$ to be $(2 n+1) \Delta r$, where $n$ is the number of considered range gates on both sides of the range gate comprising $R_{3}$.

A rain rate pattern $R(i)$ is freely defined according to the requirements of the simulated case. The rain rate is assumed to be constant within one range gate. Out of this, the DSD $N\left(D_{j}, i\right)$ is calculated for each range gate using the Marshall-Palmer standard distribution (Marshall and Palmer, 1948). Drop diameter classes $D_{j}$ from 0.15 to $6.5 \mathrm{~mm}$ with a class width of $0.05 \mathrm{~mm}$ are used here. Then, the theoretical, intrinsic reflectivity $Z(i)$, depending on DSD and backscattering cross section, is calculated. Finally, attenuated reflectivities $Z_{1}(i), Z_{2}(i)$ and $Z_{3}(h)$ - describing measurements from $R_{1}, R_{2}$ and $R_{3}$, respectively - are calculated in analogy with Eq. (1). The specific attenuation $k_{3}(i)$ required to simulate the attenuated reflectivity is derived from Eq. (3). The extinction cross section is calculated using Mie theory according to Morrison and Cross (1974), considering droplet flattening.

This study aims at analysing how the calibration accuracy depends on rainfall intensity and structure, and on the width of the interval chosen for determination of specific attenuation $k\left(s_{3}\right)$ around $\mathrm{R}_{3}$, defined by $n$. Synthetic data are simulated for two idealised rainfall patterns (homogeneous rain intensity along (Fig. 2) or featuring a maximum in the middle of (Fig. 3) the measuring path) with 15 different rainfall
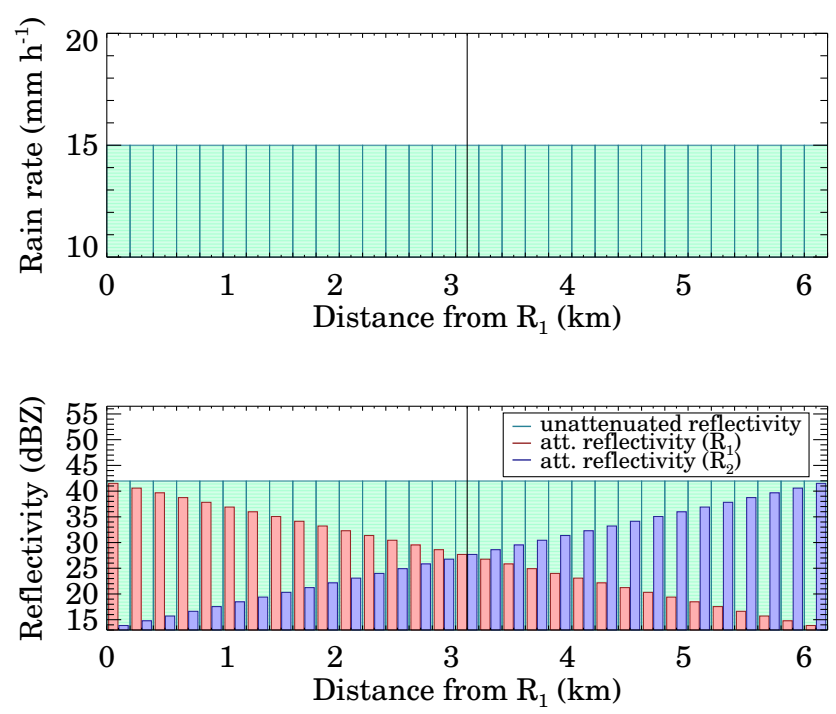

Figure 2. Idealised synthetic data simulated along the measuring path. Homogeneous precipitation pattern with a rainfall intensity of $15 \mathrm{~mm} \mathrm{~h}^{-1}$ (top panel) and corresponding reflectivity fields simulated using forward operator (bottom panel). Intrinsic reflectivity is shown in green and simulated, attenuated reflectivity in red for $\mathrm{R}_{1}$ and blue for $R_{2}$. The vertical black line marks the position of $R_{3}$.

intensities in each case according to the method described above. For these rainfall patterns calibration is performed using 12 different widths $(2 n+1) \Delta r$, with $n$ varying from 1 to 12 range gates on both sides of $R_{3}$. Hence, the sensitivity study comprises 180 different combinations of rain rates $R(i)$ and interval widths $(2 n+1) \Delta r$. In order to take into account measurement uncertainties, which are inherent to data, calibration is run in a Monte Carlo simulation with 100 repetitions for each combination, assigning random errors to the data for each of the 100 repetitions. For this the simulated radar reflectivity fields are overlaid with a Gaussian distributed noise with a standard deviation of $2 \mathrm{dBZ}$ for each range gate. For both rainfall patterns, mean and standard deviation of the correction factor $C_{3}^{-1}$ are calculated from the Monte Carlo simulation results.

In a first analysis, a homogeneous rainfall pattern is considered with rainfall intensities varying between 1 and $15 \mathrm{~mm} \mathrm{~h}^{-1}$. Mean correction factors $C_{3}^{-1}$ (Fig. 4a) are close to the expected value of 1.0 (perfectly calibrated radar), between 0.995 and 1.005 for most of the tested combinations. Higher deviations can be found at short interval widths with $1 \leq n \leq 3$ range gates and for rain rates below $5 \mathrm{~mm} \mathrm{~h}^{-1}$ (corresponding to approximately $30 \mathrm{dBZ}$ ) where values range between 0.95 and 1.25 . The stability of these results, quantified by the standard deviation of the obtained factors (Fig. 4b), increases towards higher rain rates and larger $(2 n+1) \Delta r$ as the standard deviation decreases. Starting at high values above 2.00 (3.00 for $n=1$ and lowest rain rate), which indicate a spread of more than twice the expected value of the 

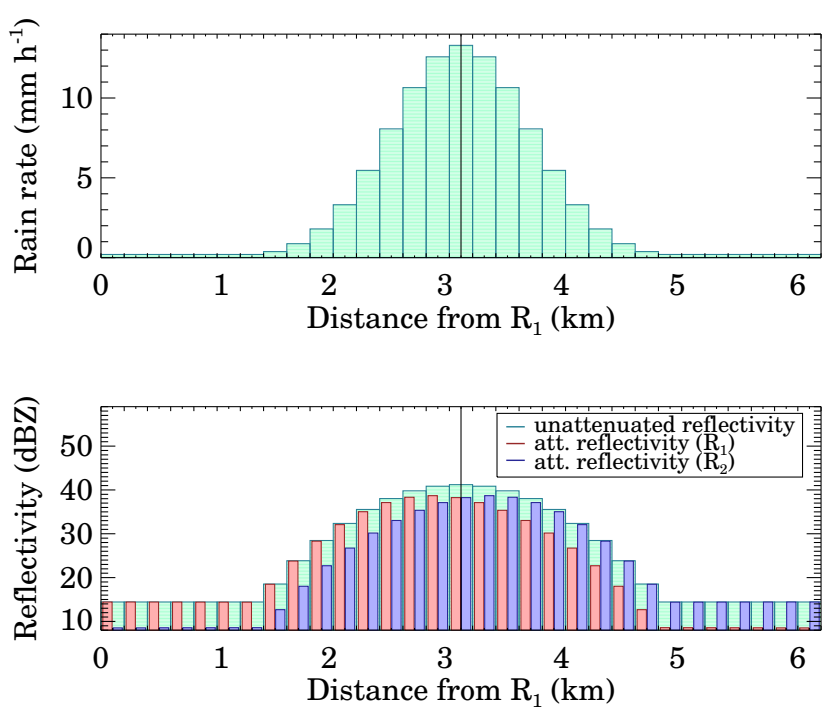

Figure 3. In analogy with Fig. 2, rain rate and simulated reflectivity fields for a precipitation pattern with a maximum intensity above $\mathrm{R}_{3}$ (maximum rain rate is $13.3 \mathrm{~mm} \mathrm{~h}^{-1}$ ).

calibration factor, the standard deviation reaches values below 0.1 for $R \geq 5 \mathrm{~mm} \mathrm{~h}^{-1}$ and $n \geq 4$. The standard deviation decreases with increasing rain rate and range gate number $n$, reaching down to 0.016 . Hence, calibration of $R_{3}$ is found to be best for high rain rates and large $n$, both having the effect of producing a clear and detectable attenuation signal along the considered path section required by the calibration method. Note that, when considering real conditions, the assumption of a homogeneous DSD within the section of interest becomes less applicable the larger the section is; i.e. the larger $n$ is chosen.

The study of the second analysed precipitation pattern, characterised by a maximum value in rain rate above the location of $R_{3}$, investigates possible impacts of precipitation maxima or minima on the calibration results. This precipitation field (Fig. 3) is created by using the shape of a normal distribution in order to define a rain rate $R(i)$ in each range gate from a maximum rain rate. The maximum rain rate (value above $R_{3}$ ) is varied between 4.0 and $13.3 \mathrm{~mm} \mathrm{~h}^{-1}$, creating 15 fields with different rainfall intensities. Figure $5 \mathrm{a}$ and $\mathrm{b}$ depict the results for averaged correction factor $C_{3}^{-1}$ and corresponding standard deviation (note that the colour scales of Figs. 5a and 4a are different). The calibration results differ from the one discussed above for homogeneous rain fields. Here, the mean calibration factor is well determined only for high rainfall intensities and small intervals $(2 n+1) \Delta r$ where it lies in the interval between 0.95 and 1.05. For high rain rates and increasing interval width, the correction factor shows a large negative bias with values down to 0.313 , which represents an error of almost $70 \%$ in the calibration. The standard deviation is less dependent on rainfall intensity. Stable results (standard deviations below 0.1) are achieved for $n>6$. A slight tendency toward lower standard deviations at higher rain rates is still visible. This obtained bias in the correction factor $C_{3}^{-1}$ can be explained by the shape of the precipitation field. Maximum rain rate above $\mathrm{R}_{3}$ induces an observed (attenuation-corrected) DSD, and consequently attenuation, at the position $s_{3}$ that is not representative for the whole interval $(2 n+1) \Delta r$. Attenuation corrected specific attenuation $k_{3}\left(s_{3}\right) \cdot \exp \left(2 k\left(s_{3}\right) h\right)$ calculated from DSD measurements of $\mathrm{R}_{3}$ (Eq. 5) is then higher than specific attenuation $k\left(s_{3}\right)$ calculated from measurements of $\mathrm{R}_{1}$ and $\mathrm{R}_{2}$ along the considered interval (Eq. 11), which assumes a constant, averaged specific attenuation. Consequently, correction factor $C_{3}^{-1}$ is erroneously found to be smaller than 1.0 (Eq. 7). The opposite effect occurs in the case of a minimum in the rainfall intensity above $\mathrm{R}_{3}$.

\section{Test on synthetic data with realistic precipitation patterns}

Since the presented method is found to be valid when using idealised, smooth precipitation patterns, a further study is realised based on data with realistic rainfall patterns. For this purpose, measured MRR data from a network installed at the Meteorological Observatory Lindenberg (MOL) operated by the German Meteorological Service (DWD) are used in order to create synthetic data with a realistic texture as given by measurements. The network fits the conditions introduced in Sect. 2, and data were recorded between the beginning of May and end of June 2013. Within this period 15 rainfall events, comprising $422010 \mathrm{~s}$ time steps in total, are chosen for testing the calibration method. For slanted devices $R_{1}$ and $R_{2}$, reflectivity is calculated directly by integration of the power spectrum, as done before by e.g. Maahn and Kollias (2012) and Kneifel et al. (2011). The values given by the standard MRR software are derived from the measured DSD and are only valid for vertically pointing MRRs.

In order to obtain realistic precipitation fields, reflectivity measurements from both horizontally oriented MRRs are used to generate synthetic, intrinsic reflectivity fields along the path. These synthetic, intrinsic reflectivity fields are created by comparing and combining measurements from $R_{1}$ and $R_{2}$ such that the highest reflectivity value of both is selected in each range gate. Using measurements from just one MRR would yield synthetic, intrinsic reflectivity fields showing a systematic decrease in reflectivity toward one side of the measuring path, as an artefact of attenuation present in real measurements. From the obtained reflectivity fields, rain rate and synthetic, attenuated reflectivity for all three radars are simulated according to the procedure described in Sect. 3. All devices are still considered to be perfectly calibrated for this analysis, yielding a correction factor $C_{3}^{-1}$ of 1.0.

Since two characteristics of reflectivity fields have been detected to be disadvantageous for calibration in Sect. 3 (high 


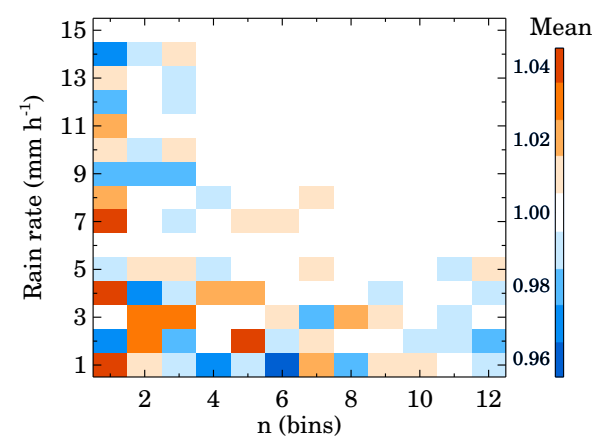

(a)

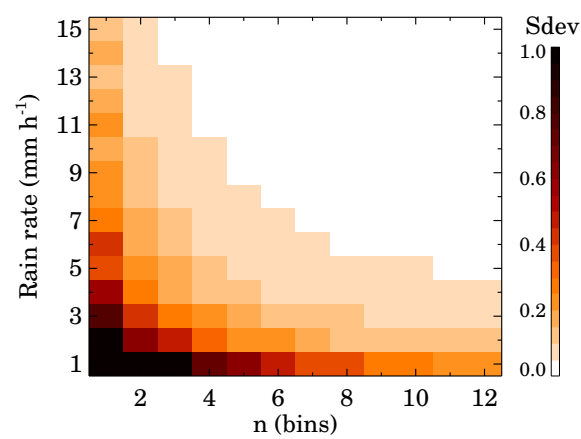

(b)

Figure 4. Mean (a) and standard deviation (b) of the correction factor $C_{3}^{-1}$ for homogeneous precipitation patterns, calculated from Monte Carlo simulations including 100 repetitions with random measuring error. The Monte Carlo simulations are performed for combinations of 15 different intensities of the homogeneous precipitation field and 12 considered interval widths $(2 n+1) \Delta r$ for attenuation determination above $\mathrm{R}_{3}$.

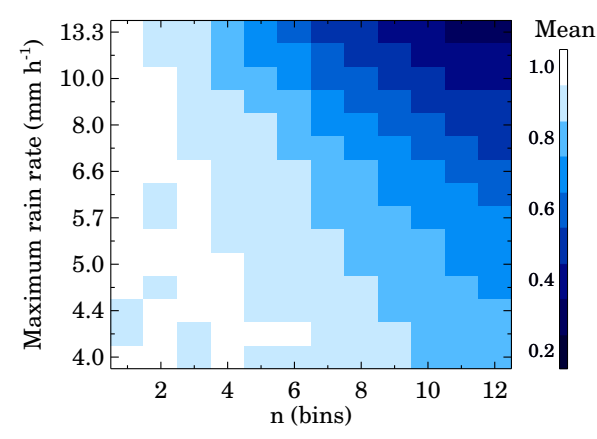

(a)

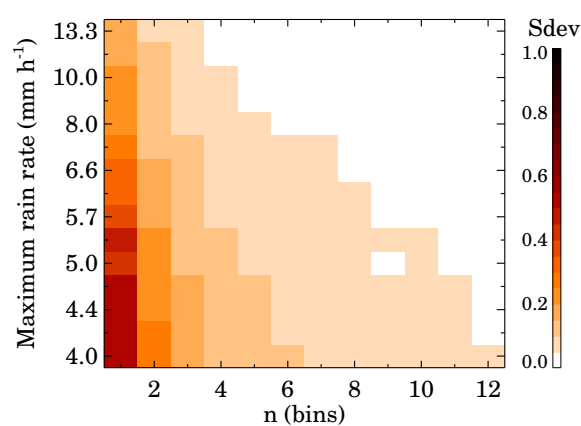

(b)

Figure 5. Mean (a) and standard deviation (b) of the correction factor $C_{3}^{-1}$ for Gaussian-shaped rain fields, calculated from Monte Carlo simulations including 100 repetitions with random measuring error. The Monte Carlo simulations are performed for combinations of 15 different rainfall intensities and 12 considered interval widths $(2 n+1) \Delta r$ for attenuation determination above $\mathrm{R}_{3}$.

heterogeneity and low rain rates along the measuring section), the simulated reflectivity fields are filtered using two parameters in order to remove unsuited cases. The prerequisite for a good calibration is an attenuation effect strong enough to be detected reliably. Therefore, the rainfall intensity along the measuring path has to be high enough to achieve the required signal extinction. Averaged reflectivity along the measuring path is calculated and a threshold is set to $30 \mathrm{dBZ}$. Furthermore, strong inhomogeneities, evidence of high noise or disturbances in the measurements, can falsify calibration. Therefore these data are filtered out. This is done by using the texture of the reflectivity in $\mathrm{dBZ}$ (TDBZ) according to Hubbert et al. (2009):

$\mathrm{TDBZ}=\sum_{i}(\mathrm{dBZ}(i)-\mathrm{dBZ}(i-1))^{2} \cdot \frac{1}{I}$

TDBZ is an indicator for fluctuation of reflectivity along the path calculated by summing up the squared differences in reflectivity between adjacent range gates $i$. Here, $I$ represents the total number of range gates considered for the TDBZ calculation, which has to be chosen appropriately. A TDBZ threshold over the considered $I=11$ range gates in the middle of the measuring path is set to $1.4 \mathrm{dBZ}^{2}$. This threshold is defined by studying the quality of calibration results running a Monte Carlo simulation as presented in Sect. 3 for the 4220 chosen time steps. Ninety percent of the cases having at most $10 \%$ error in the determination of the correction factor and standard deviation below 1.0 have to show TDBZ lower than the threshold. After filtering with averaged rain rate and TDBZ, 3246 suited time steps remain for calibration and are used in the following. After generation and selection of synthetic reflectivity fields, calibration of $\mathrm{R}_{3}$ is performed in order to analyse the behaviour of the calibration method when applied to data showing realistic patterns. The calibration is performed once for each time step, with an added random, Gaussian-shaped fluctuation with a standard deviation of $2 \mathrm{dBZ}$ which simulates measurement uncertainties. Eleven different intervals $(2 n+1) \Delta r$ are considered, with $n$ varying from 2 to 12 ( $n=1$ was found to provide unsatisfactory results in Sect. 3). 


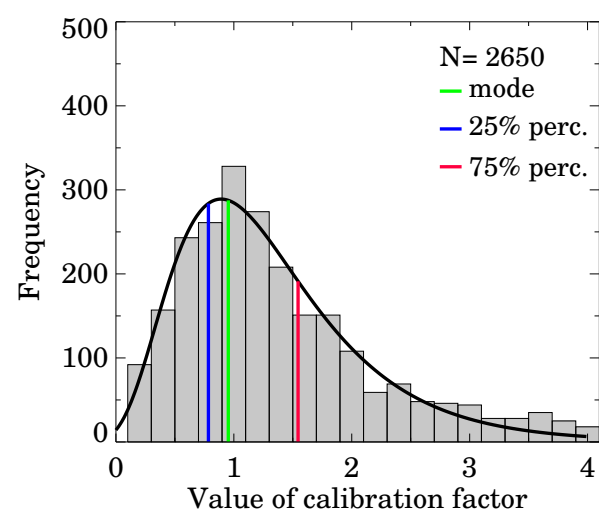

(a) $n=2$

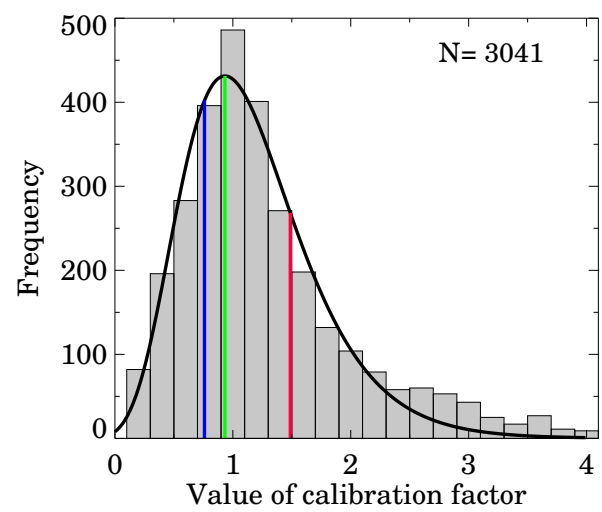

(c) $n=6$

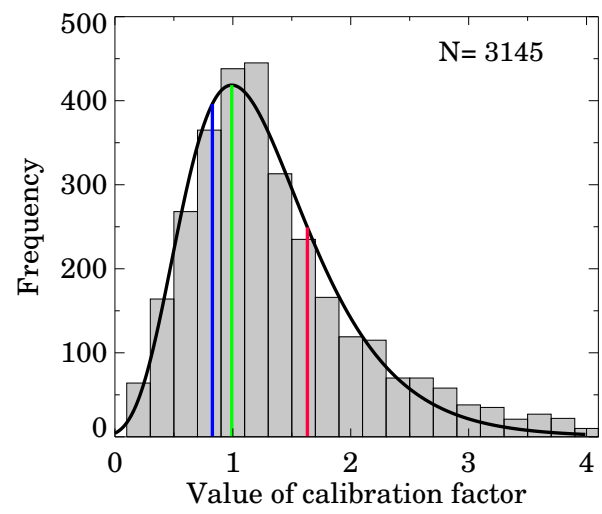

(e) $n=10$

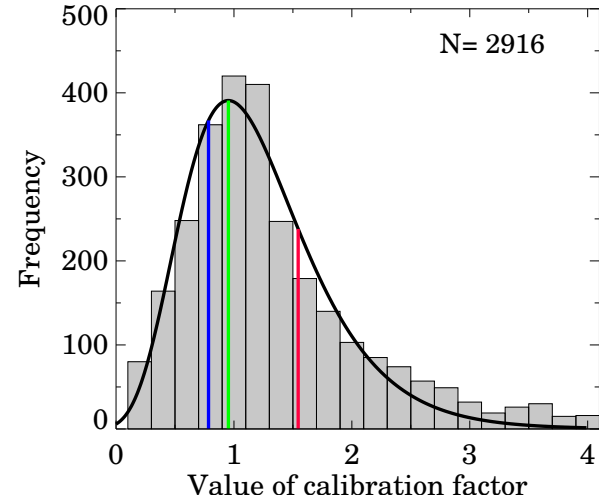

(b) $n=4$

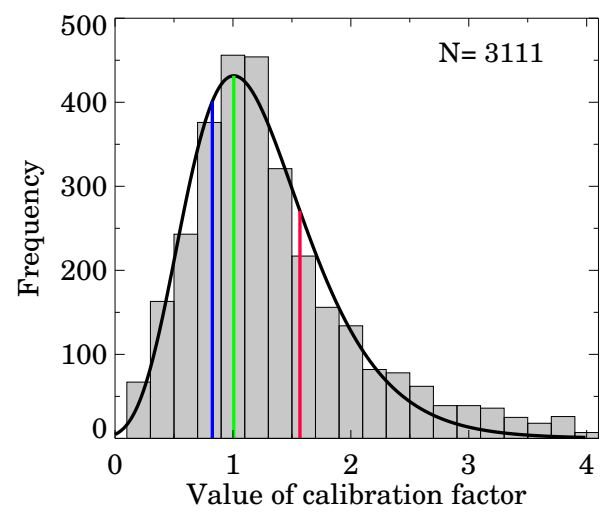

(d) $n=8$

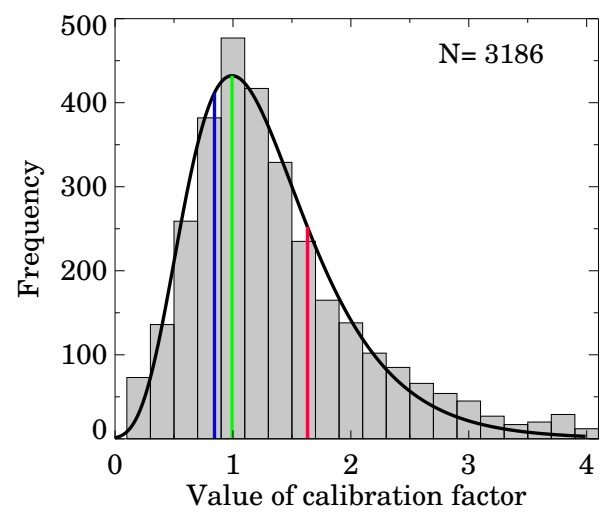

(f) $n=12$

Figure 6. Distributions of the correction factor $C_{3}^{-1}$ calculated using synthetic data with realistic precipitation patterns and fitted logarithmic normal distribution functions for six values of $n$. The total amount of considered time steps is indicated by $N$. The mode, 25 th and 75 th percentile of the fitted distributions are shown in green, blue and red, respectively.

Calibration using the selected time steps and intervals $(2 n+1) \Delta r$ does not achieve precise results in all cases. Resulting correction factors are spread over a wide range of values. In order to define a method for the determination of the wanted correction factor, distributions of obtained $C_{3}^{-1}$ are studied (Fig. 6). For each considered number of range gates $n$, the calculated correction factors are considered among 20 classes with a width of 0.2 . Time steps providing negative calibration factors are removed, resulting in a different number $N$ of remaining calibration results for the 11 different interval widths. Those negative results have no physical meaning, as corrected reflectivity fields would then also ap- 
Table 1. Mode, 25th and 75th percentiles and interquartile range calculated from the logarithmic normal distribution functions fitted to the results for $C_{3}^{-1}$ for $2 \leq n \leq 12$ (rounded values). Calibration was performed using synthetic data with realistic precipitation structures.

\begin{tabular}{lccccccccccc}
\hline & $n=2$ & $n=3$ & $n=4$ & $n=5$ & $n=6$ & $n=7$ & $n=8$ & $n=9$ & $n=10$ & $n=11$ & $n=12$ \\
\hline Mode & 0.89 & 0.91 & 0.95 & 0.93 & 0.93 & 0.96 & 1.01 & 1.00 & 0.99 & 1.01 & 0.99 \\
25th percentile & 0.76 & 0.76 & 0.78 & 0.78 & 0.76 & 0.78 & 0.83 & 0.83 & 0.83 & 0.83 & 0.84 \\
75th percentile & 1.72 & 1.62 & 1.54 & 1.53 & 1.49 & 1.47 & 1.57 & 1.63 & 1.63 & 1.60 & 1.63 \\
\hline Interquartile range & 0.97 & 0.86 & 0.76 & 0.75 & 0.73 & 0.69 & 0.74 & 0.80 & 0.81 & 0.77 & 0.79 \\
\hline
\end{tabular}

pear to be negative (Eq. 2). According to Eq. (7) negative correction factors appear if $k\left(s_{3}\right) \leq 0$. Since this study is based on synthetic data, as described above, non-physical, negative specific attenuation values are an artefact of the added random noise creating strong inhomogeneities and missed by the TDBZ filter. These cases should not be considered. Distributions of the correction factor have their maximum within the class including 1.0 (except for $n=10$ ), which is the expected correction factor for a perfectly calibrated radar, and are positively skewed. As discrete distributions only allow for the estimation of a median within the given resolution, a shifted logarithmic normal distribution function is fitted to the discrete distribution in order to provide more precise results. The most probable value of the wanted correction factor is given by the mode of the distribution function, and thus the most frequently calculated value. In order to describe the width of the distribution, which describes the accuracy of calibration, the interquartile range (describing the distance between the 25th and 75th percentile) is also considered (Table 1). Obtained distribution functions have the lowest interquartile range for $4 \leq n \leq 8$, indicating the most stable results for these settings. The widest spread of the calculated correction factor is found using $n=2$. The error in the estimation of the correction factor, knowing the true value is 1.0 , reaches $11 \%$ for $n=2$ and is lowest for $n \geq 8$, where $C_{3}^{-1}$ is determined by the mode with an error of only $1 \%$. It is exactly 1.0 for $n=9$. These results suggest the possibility to achieve satisfactory calibration results when applying the presented calibration method to carefully chosen data and settings. Since a considered interval $(2 n+1) \Delta r$ with $4 \leq n \leq 8$ yields the most stable results, and the most accurate results are achieved with $n \geq 8, n=8$ is possibly the most appropriate setting for calibration in the network considered here.

\section{Summary and conclusions}

A novel method for absolute radar calibration with respect to reflectivity and without reference device is analysed in this study. The method is first tested using synthetic data with idealised, smooth precipitation patterns to prove the validity of the concept. Homogeneous and Gaussian-shaped precipitation patterns are analysed and calibration performed consid- ering simulated data with a measuring uncertainty of $2 \mathrm{dBZ}$. Furthermore, different rainfall intensities and interval widths $(2 n+1) \Delta r$ for determination of the attenuation $k\left(s_{3}\right)$ are taken into account. Calibration using homogeneous precipitation patterns yields precise results. Mean correction factor $C_{3}^{-1}$ for $\mathrm{R}_{3}$ takes values between 0.995 and 1.005 , except from calibration at rain rates below $5 \mathrm{~mm} \mathrm{~h}^{-1}$ and with $n \leq 3$. This represents an error of $0.5 \%$ in the determination of the true calibration factor, which is 1.0 for a perfectly calibrated radar as simulated in this analysis. The standard deviation, expressing the stability of the procedure, stays below $10 \%$ for $R \geq 5 \mathrm{~mm} \mathrm{~h}^{-1}$ and $n \geq 4$. It decreases towards higher rain rates and larger interval widths, reaching down to under $2 \%$. Inhomogeneous precipitation patterns, featuring higher (lower) rainfall intensity than average above $\mathrm{R}_{3}$, reveal one weakness of the method. Due to less likely high (low) rain rates above $\mathrm{R}_{3}$, its measurements are erroneously corrected toward lower (higher) average reflectivity values, inducing a negative (positive) bias in the calculated correction factor. This bias is stronger the higher the rain rate and the larger the interval along which the attenuation is determined. Since a minimum in rainfall intensity above $R_{3}$ is likely to occur as often as a maximum, inducing a positive bias, this effect will be cancelled out when averaging over numerous rainfall events.

The theoretical validity of the presented absolute calibration method has been proved for adequate precipitation patterns. Ideal cases are preferably homogeneous, intense rainfall along the measuring path, leading to attenuation strong enough to be determined reliably.

Since these first promising results are obtained focusing on idealised synthetic data, a further study is done with synthetic data featuring realistic structures. Those structures are taken from reflectivity measurements from a real deployment of MRRs following the concept introduced in Sect. 2. Considered time steps are filtered using averaged reflectivity along the measuring path and texture of the reflectivity field TDBZ in order to guarantee high attenuation and smooth measurement structures. Calibration over a 3246 sample of filtered time steps of reflectivity measurements leads to distributions of calculated correction factors which can be described using shifted logarithmic normal distribution functions. These functions are fitted to the obtained, results and the mode is 
calculated in order to describe the sought-after correction factor for each considered $n$. Here, the calibration results vary between 0.89 and 1.01 , i.e. up to $11 \%$ error. The best results are achieved using $n=8$.

Future analysis applying the method to selected network data, including comparison with reference devices and established calibration methods, have to be performed in order to prove its applicability in practice. Some problems will arise from using real, measured data, and their impact on calibration accuracy must then be evaluated. Regarding the network setup, accurate alignment of $R_{1}$ and $R_{2}$ and the height of the measuring path should be considered in order to ensure consistent measurements and minimise beam blockage from the ground. Since measuring volumes are different, the vertical variability of DSD can induce errors when comparing measurements in strongly inhomogeneous cases. Furthermore, errors in the DSD measurements from $\mathrm{R}_{3}$, caused mainly by vertical winds, should be analysed. In order to obtain precise calibration, it will be important to improve the criteria to select suitable data. Beside testing the presented parameters (rain intensity and TDBZ for rain homogeneity), removing convective events (with strong turbulence) and time steps with vertically strongly varying DSD (making use of measurements from $\mathrm{R}_{3}$ ) is probably necessary. The sensitivity of calibration with respect to the integration time, finding the optimum between minimising noise and still resolving rainfall variability, also needs further study.

Nevertheless, the fundamental analysis of the presented novel method for absolute radar calibration in a network proves its theoretical validity. The method could offer great opportunities for absolute calibration of radar networks operating in strongly attenuated frequency ranges (e.g. K and $\mathrm{X}$ band), providing accurate and comparable data for application.

Acknowledgements. Thanks are due to Ulrich Görsdorf and the team of the Meteorological Observatory Lindenberg (MOL) of the German Meteorological Service (DWD) for supporting the project and enabling the setup of a micro rain radar network at MOL as well as to Hans Münster for installing and maintaining the installed devices.

This work is part of the Precipitation and Attenuation Estimates from a High-Resolution Weather Radar Network (PATTERN) project and is funded by the Deutsche Forschungsgemeinschaft (grant AM308/3-1).

Edited by: G. Vulpiani

\section{References}

Atlas, D., Srivastava, R. C., and Sekhon, R. S.: Doppler radar characteristics of precipitation at vertical incidence, Rev. Geophys., 11, 1-35, 1973.

Bringi, V. and Chandrasekar, V.: Polarimetric Doppler Weather Radar: Principles and Applications, Cambridge University Press, 2001.

Chandrasekar, V. and Jayasumana, A.: Radar design and management in a networked environment, in: Technologies, Protocols, and Services for Next-Generation Internet, Vol. 4527 of Proceedings of the Society of Photo-Optical Instrumentation Engineers (SPIE), SPIE; Colorado Photon Ind Assoc, Conference on Technologies, Protocols, and Services for Next-Generation Internet, Denver, CO, 21-23 August 2001, 142-147, 2001.

Corral, C., Velasco, D., Forcadell, D., Sempere-Torres, D., and Velasco, E.: Advances in radar-based flood warning systems. The EHIMI system and the experience in the Besos flashflood pilot basin, Flood Risk Manage. Res. Pract., 1295-1303, doi:10.1201/9780203883020.ch153, 2008.

Creutin, J. and Borga, M.: Radar hydrology modifies the monitoring of flash-flood hazard, Hydrol. Process., 17, 1453-1456, 2003.

Crum, T., Saffle, R., and Wilson, J.: An update on the NEXRAD program and future WSR-88D support to operations, Weather Forecast., 13, 253-262, 1998.

Delrieu, G., Braud, I., Berne, A., Borga, M., Boudevillain, B., Fabry, F., Freer, J., Gaume, E., Nakakita, E., Seed, A., Tabary, P., and Uijlenhoet, R.: Weather radar and hydrology Preface, Adv. Water Resour., 32, 969-974, 2009.

Einfalt, T., Arnbjerg-Nielsen, K., Golz, C., Jensen, N., Quirmbach, M., Vaes, G., and Vieux, B.: Towards a roadmap for use of radar rainfall data in urban drainage, J. Hydrol., 299, 186-202, 2004.

Gires, A., Tchiguirinskaia, I., Schertzer, D., Schellart, A., Berne, A., and Lovejoy, S.: Influence of small scale rainfall variability on standard comparison tools between radar and rain gauge data, Atmos. Res., 138, 125-138, 2014.

Hubbert, J. C., Dixon, M., and Ellis, S. M.: Weather radar ground clutter, Part II: Real-time identification and filtering, J. Atmos. Ocean. Tech., 26, 1181-1197, doi:10.1175/2009JTECHA1160.1, 2009.

Hunter, S.: WSR-88D radar rainfall estimation: Capabilities, limitations and potential improvements, Natl. Wea. Dig., 20, 26-38, 1996.

Jaffrain, J., and Berne, A.: Influence of the Subgrid Variability of the Raindrop Size Distribution on Radar Rainfall Estimators, J. Appl. Meteorol. Clim., 51, 780-785, 2012a.

Jaffrain, J., and Berne, A.: Quantification of the Small-Scale Spatial Structure of the Raindrop Size Distribution from a Network of Disdrometers, J. Appl. Meteorol. Clim., 51, 1558-8424, 2012 b.

Jensen, N. and Pedersen, L.: Spatial variability of rainfall: variations within a single radar pixel, 6th International Workshop on Precipitation in Urban Areas, Pontresina, Switzerland, 4-7 December 2003, Atmos. Res., 77, 269-277, 2005.

Kneifel, S., Maahn, M., Peters, G., and Simmer, C.: Observation of snowfall with a low-power FM-CW K-band radar (Micro Rain Radar), Meteorol. Atmos. Phys., 113, 75-87, 2011.

Krajewski, W. and Smith, J.: Radar hydrology: rainfall estimation, Adv. Water Resour., 25, 1387-1394, 2002. 
Krajewski, W., Ciach, G., and Habib, E.: An analysis of smallscale rainfall variability in different climatic regimes, Hydrolog. Sci. J., 48, 151-162, doi:10.1623/hysj.48.2.151.44694, 2003.

Lee, G.W., and Zawadzki, I.: Radar calibration by gage, disdrometer, and polarimetry: Theoretical limit caused by the variability of drop size distribution and application to fast scanning operational radar data, J. Hydrol., 328, 83-97, 2006.

Lengfeld, K., Clemens, M., Münster, H., and Ament, F.: Performance of high-resolution X-band weather radar networks the PATTERN example, Atmos. Meas. Tech., 7, 4151-4166, doi:10.5194/amt-7-4151-2014, 2014.

Lim, S., Chandrasekar, V., Lee, P., and Jayasumana, A. P.: Real-time implementation of a network-based attenuation correction in the CASA IP1 testbed, J. Atmos. Ocean. Tech., 28, 197-209, 2011.

Maahn, M. and Kollias, P.: Improved Micro Rain Radar snow measurements using Doppler spectra post-processing, Atmos. Meas. Tech., 5, 2661-2673, doi:10.5194/amt-5-2661-2012, 2012.

Mandapaka, P. V., Lewandowski, P., Eichinger, W. E., and Krajewski, W. F.: Multiscaling analysis of high resolution spacetime lidar-rainfall, Nonlin. Processes Geophys., 16, 579-586, doi:10.5194/npg-16-579-2009, 2009.

Marshall, J. and Palmer, W.: The distribution of raindrops with size, J. Meteorol., 5, 165-166, 1948.

McLaughlin, D., Pepyne, D., Chandrasekar, V., Philips, B., Kurose, J., Zink, M., Droegemeier, K., Cruz-Pol, S., Junyent, F., Brotzge, J., Westbrook, D., Bharadwaj, N., Wang, Y., Lyons, E., Hondl, K., Liu, Y., Knapp, E., Xue, M., Hopf, A., Kloesel, K., DeFonzo, A., Kollias, P., Brewster, K., Contreras, R., Dolan, B., Djaferis, T., Insanic, E., Frasier, S., and Carr, F.: Shortwavelength technology and the potential for distributed networks of small radar systems, B. Am. Meteorol. Soc., 90, 1797-1817, 2009.

Moreau, E., Testud, J., and Le Bouar, E.: Rainfall spatial variability observed by X-band weather radar and its implication for the accuracy of rainfall estimates, Adv. Water Resour., 32, 1011-1019, 2009.

Morrison, J. and Cross, M.: Scattering of a plane electromagneticwave by axisymmetric raindrops, Bell Syst. Tech. J., 53, 9551019, 1974.

Nielsen, J. E., Jensen, N. E., and Rasmussen, M. R.: Calibrating LAWR weather radar using laser disdrometers, Atmos. Res., 122, 165-173, 2013.

Pedersen, L., Jensen, N., Christensen, L., and Madsen, H.: Quantification of the spatial variability of rainfall based on a dense network of rain gauges, Atmos. Res., 95, 441-454, 2010a.

Pedersen, L., Jensen, N., and Madsen, H.: Calibration of Local Area Weather Radar-Identifying significant factors affecting the calibration, Atmos. Res., 97, 129-143, 2010b.

Peleg, N., Ben-Asher, M., and Morin, E.: Radar subpixel-scale rainfall variability and uncertainty: lessons learned from observations of a dense rain-gauge network, Hydrol. Earth Syst. Sci., 17, 2195-2208, doi:10.5194/hess-17-2195-2013, 2013.
Peters, G., Fischer, B., and Andersson, T.: Rain observations with a vertically looking Micro Rain Radar (MRR), Boreal Environ. Res., 7, 353-362, 2002.

Peters, G., Fischer, B., Münster, H., Clemens, M., and Wagner, A.: Profiles of raindrop size distributions as retrieved by microrain radars, J. Appl. Meteorol., 44, 1930-1949, 2005.

Picciotti, E., Marzano, F. S., Anagnostou, E. N., Kalogiros, J., Fessas, Y., Volpi, A., Cazac, V., Pace, R., Cinque, G., Bernardini, L., De Sanctis, K., Di Fabio, S., Montopoli, M., Anagnostou, M. N., Telleschi, A., Dimitriou, E., and Stella, J.: Coupling X-band dualpolarized mini-radars and hydro-meteorological forecast models: the HYDRORAD project, Nat. Hazards Earth Syst. Sci., 13, 1229-1241, doi:10.5194/nhess-13-1229-2013, 2013.

Quintero, F., Sempere-Torres, D., Berenguer, M., and Baltas, E.: A scenario-incorporating analysis of the propagation of uncertainty to flash flood simulations, J. Hydrol., 460, 90-102, 2012.

Salazar, J., Hopf, A., Contreras, R., Philips, B., Knapp, E., McLaughlin, D., Brotzge, J., and Brewster, K.: Coverage comparison of short range radar networks vs. conventional weather radars: case study in the Northwestern United States, in: 2009 IEEE International Geoscience and Remote Sensing Symposium (IGARSS 2009), 12-17 July 2009, Cape Town, South Africa, 964-967, 2009.

Sebastianelli, S., Russo, F., Napolitano, F., and Baldini, L.: On precipitation measurements collected by a weather radar and a rain gauge network, Nat. Hazards Earth Syst. Sci., 13, 605-623, doi:10.5194/nhess-13-605-2013, 2013.

Thorndahl, S. and Rasmussen, M.: Marine X-band weather radar data calibration, 8th International Workshop on Precipitation in Urban Areas, St. Moritz, Switzerland, 10-13 December 2009, Atmos. Res., 103, 33-44, 2012.

Trabal, J., Colom-Ustariz, J., Cruz-Pol, S., Pablos-Vega, G., and McLaughlin, D.: Remote sensing of weather hazards using a lowcost and minimal infrastructure off-the-grid weather radar network, IEEE T. Geosci. Remote, 51, 2541-2555, 2013.

Ulbrich, C. and Lee, L.: Rainfall measurement error by WSR-88D radars due to variations in $Z-R$ law parameters and the radar constant, J. Atmos. Ocean. Tech., 16, 1017-1024, 1999.

Van Baelen, J., Pointin, Y., Wobrock, W., Flossmann, A., Peters, G., Tridon, F., and Planche, C.: Precipitation and microphysical studies with a low cost high resolution X-band radar: an innovative project prospective, Adv. Geosci., 20, 25-32, doi:10.5194/adgeo20-25-2009, 2009.

Villarini, G. and Krajewski, W.: Review of the different sources of uncertainty in single polarization radar-based estimates of rainfall, Surv. Geophys., 31, 107-129, 2010.

Vuerich, E., Monesi, C., Lanza, L. G., Stagi, L., and Lanzinger, E.: WMO field intercomparison of rainfall intensity gauges (Vigna di Valle, Italy, October 2007-April 2009), WMO IOM99, WMOTD 1504, CIMO IOM Reports, WMO, 2009. 